\title{
Robust Stability and Performance Analysis of 2D Mixed Continuous-Discrete-Time Systems with Uncertainty
}

\author{
Graziano Chesi $^{1}$ and Richard H. Middleton ${ }^{2}$ \\ ${ }^{1}$ Department of Electrical and Electronic Engineering \\ The University of Hong Kong \\ Hong Kong \\ 2 School of Electrical Engineering and Computer Science \\ The University of Newcastle \\ Australia
}

\begin{abstract}
This paper investigates 2D mixed continuous-discrete-time systems whose coefficients are polynomial functions of an uncertain vector constrained into a semialgebraic set. It is shown that a nonconservative linear matrix inequality (LMI) condition for ensuring robust stability can be obtained by introducing complex Lyapunov functions depending polynomially on the uncertain vector and a frequency. Moreover, it is shown that nonconservative LMI conditions for establishing upper bounds of the robust $\mathcal{H}_{\infty}$ and $\mathcal{H}_{2}$ norms can be obtained by introducing analogous Lyapunov functions depending rationally on the frequency. Some numerical examples illustrate the proposed methodology.
\end{abstract}

Keyword: 2D Systems, Uncertainty, Robust stability, Robust performance.

\section{Introduction}

The study of 2D mixed continuous-discrete-time systems has a long history, with some early works such as $[1,2]$ introducing basic models, systems theory and stability properties. Applications of these systems can be found in 
repetitive processes [3], disturbance propagation in vehicle platoons [4], and irrigation channels [5].

Researchers have investigated several fundamental properties of 2D mixed continuous-discrete-time systems, in particular stability, for which key contributions include [3,6-9]. Other fundamental properties that have been investigated in $2 \mathrm{D}$ mixed continuous-discrete-time systems are the $\mathcal{H}_{\infty}$ and $\mathcal{H}_{2}$ norms, for which the contributions include [10-12] where conditions based on linear matrix inequalities (LMIs) have been provided. The reader is also referred to $[13,14]$ for related contributions in other areas of $2 \mathrm{D}$ systems.

However, these conditions cannot be used whenever the matrices of the model are affected by uncertainty. In fact, in such a case, one should repeat the existing conditions addressing the uncertainty-free case for all the admissible values of the uncertainty. Clearly, this is impossible since the number of values in a continuous set is infinite and one cannot just consider a finite subset of values such as the vertices in the case this set is a polytope. It should be mentioned that various methods have been proposed in the literature for stability and performance analysis of 1D systems affected by uncertainty, such as [15-21].

This paper investigates 2D mixed continuous-discrete-time systems affected by uncertainty. It is supposed that the coefficients of the systems are polynomial functions of an uncertain vector constrained into a semialgebraic set. It is shown that an LMI condition for ensuring robust stability can be obtained by introducing complex Lyapunov functions depending polynomially on the uncertain vector and a frequency. Moreover, it is shown that LMI conditions for establishing upper bounds of the robust $\mathcal{H}_{\infty}$ and $\mathcal{H}_{2}$ norms can be obtained by introducing analogous Lyapunov functions depending rationally on the frequency. These LMI conditions are sufficient for any chosen degree of the complex Lyapunov functions, and also necessary for a sufficiently large degree of these functions under some mild assumptions on the set of admissible uncertainties. The LMI conditions proposed in this paper exploit Putinar's Positivstellensatz [22], which allows one to investigate positivity of a polynomial over a semialgebraic set by using polynomials that can be written as sums of squares of polynomials (SOS). Some numerical examples illustrate the proposed methodology.

This paper extends the preliminary conference papers $[23,24]$ by showing that the LMI condition for determining the robust $\mathcal{H}_{\infty}$ norm is nonconservative (Theorem 3) and by adding the investigation of the robust $\mathcal{H}_{2}$ norm (Section 5).

The paper is organized as follows. Section 2 provides the problem formulation and some preliminaries about SOS matrix polynomials. Section 3 
investigates the robust exponential stability. Section 4 addresses the robust $\mathcal{H}_{\infty}$ norm. Section 5 addresses the robust $\mathcal{H}_{2}$ norm. Section 6 presents some illustrative examples. Section 7 concludes the paper with some final remarks. Lastly, the appendices report some additional results.

\section{Preliminaries}

\subsection{Problem Formulation}

The notation is as follows. The real and complex number sets are denoted by $\mathbb{R}$ and $\mathbb{C}$. The imaginary unit is $j$. The symbol $I$ denotes the identity matrix (of size specified by the context). The notations $\operatorname{Re}(\cdot), \operatorname{Im}(\cdot)$ and $|\cdot|$ denote the real part, imaginary part and magnitude. The Euclidean norm and the $\mathcal{L}_{2}$ norm are denoted by $\|\cdot\|_{2}$ and $\|\cdot\|_{\mathcal{L}_{2}}$. The adjoint, determinant, null space and trace are denoted by $\operatorname{adj}(\cdot), \operatorname{det}(\cdot), \operatorname{ker}(\cdot)$ and trace $(\cdot)$. The sign is denoted by $\operatorname{sgn}(\cdot)$. The notation $A \otimes B$ denotes the Kronecker product of $A$ and $B$. The complex conjugate, transpose and complex conjugate transpose of $A$ are denoted by $\bar{A}, A^{T}$ and $A^{H}$. We say that a complex matrix $A$ is Hermitian if $A^{H}=A$. The symbol $\star$ denotes corresponding blocks in Hermitian matrices. The notations $A>0$ and $A \geq 0$ denote Hermitian positive definite and Hermitian positive semidefinite matrix $A$. The degree is denoted by $\operatorname{deg}(\cdot)$.

Let us consider the 2D mixed continuous-discrete-time system with uncertainty described by

$$
\left\{\begin{aligned}
\frac{d}{d t} x_{c}(t, k)= & A_{c c}(p) x_{c}(t, k)+A_{c d}(p) x_{d}(t, k) \\
& +B_{c}(p) u(t, k) \\
x_{d}(t, k+1)= & A_{d c}(p) x_{c}(t, k)+A_{d d}(p) x_{d}(t, k) \\
& +B_{d}(p) u(t, k) \\
y(t, k)= & C_{c}(p) x_{c}(t, k)+C_{d}(p) x_{d}(t, k) \\
& +D(p) u(t, k)
\end{aligned}\right.
$$

where $x_{c} \in \mathbb{R}^{n_{c}}$ and $x_{d} \in \mathbb{R}^{n_{d}}$ are the continuous and discrete states, the scalars $t, k \in \mathbb{R}$ are independent variables, $u \in \mathbb{R}^{n_{u}}$ and $y \in \mathbb{R}^{n_{y}}$ are the input and output, $p \in \mathbb{R}^{q}$ is a time-invariant uncertain vector, and the matrices $A_{c c}: \mathbb{R}^{q} \rightarrow \mathbb{R}^{n_{c} \times n_{c}}, A_{c d}: \mathbb{R}^{q} \rightarrow \mathbb{R}^{n_{c} \times n_{d}}, A_{d c}: \mathbb{R}^{q} \rightarrow \mathbb{R}^{n_{d} \times n_{c}}, A_{d d}:$ $\mathbb{R}^{q} \rightarrow \mathbb{R}^{n_{d} \times n_{d}}, B_{c}: \mathbb{R}^{q} \rightarrow \mathbb{R}^{n_{c} \times n_{u}}, B_{d}: \mathbb{R}^{q} \rightarrow \mathbb{R}^{n_{d} \times n_{u}}, C_{c}: \mathbb{R}^{q} \rightarrow \mathbb{R}^{n_{y} \times n_{c}}$, $C_{d}: \mathbb{R}^{q} \rightarrow \mathbb{R}^{n_{y} \times n_{d}}$ and $D: \mathbb{R}^{q} \rightarrow \mathbb{R}^{n_{y} \times n_{u}}$ are polynomial functions of degree not greater than $d_{\text {sys }}$. 
It is supposed that $p$ is constrained as

$$
p \in \mathcal{P}
$$

where $\mathcal{P}$ is the semialgebraic set

$$
\mathcal{P}=\left\{p \in \mathbb{R}^{q}: a_{i}(p) \geq 0 \forall i=1 \ldots, n_{a}\right\}
$$

and $a_{i}(p) i=1, \ldots, n_{a}$, are polynomials. No assumption is introduced on these polynomials at this stage except that $\mathcal{P}$ must be nonempty (further assumptions will be introduced on these polynomials with Definition 2 in Section 3, which will be exploited in Theorems 2, 3 and 5). Let us observe that $\mathcal{P}$ can represent a number of sets typically used to model uncertain systems, for instance:

1) hyper-ellipsoids of the form $\left\{p \in \mathbb{R}^{q}: p^{T} A p \leq 1\right\}$ where $A>0$ by choosing $n_{a}=1$ and $a_{1}(p)=1-p^{T} A p$;

2) hyper-rectangles of the form $\left\{p \in \mathbb{R}^{q}: p_{i} \in\left[p_{i}^{-}, p_{i}^{+}\right], i=1, \ldots, q\right\}$ where $p_{i}^{-}, p_{i}^{+} \in \mathbb{R}$, by choosing $n_{a}=q$ and $a_{i}(p)=\left(p_{i}^{-}-p_{i}\right)\left(p_{i}-p_{i}^{+}\right)$;

3) polytopes of the form $\left\{p \in \mathbb{R}^{q}: v_{i}^{T} p \leq w_{i}, i=1, \ldots, l\right\}$ where $v_{i} \in \mathbb{R}^{q}$ and $w_{i} \in \mathbb{R}$, by choosing $n_{a}=l$ and $a_{i}(p)=w_{i}-v_{i}^{T} p$.

Extending the classical definition of exponential stability of 2D mixed continuous-discrete-time systems [25], we say that the system (1)-(3) is robustly exponentially stable if, for a null input $u(t, k)$, there exist $\beta>0$ and $\gamma>0$ such that

$$
\left\|\left(\begin{array}{l}
x_{c}(t, k) \\
x_{d}(t, k)
\end{array}\right)\right\|_{2} \leq \beta \varrho e^{-\gamma \min \{t, k\}}
$$

for all $t \geq 0$ and $k \geq 0$, for all initial conditions $x_{c}(0, k)$ and $x_{d}(t, 0)$, and for all $p \in \mathcal{P}$, where

$$
\left\{\begin{aligned}
\varrho & =\max \left\{\varrho_{c}, \varrho_{d}\right\} \\
\varrho_{c} & =\sup _{k \geq 0}\left\|x_{c}(0, k)\right\|_{2} \\
\varrho_{d} & =\sup _{t \geq 0}\left\|x_{d}(t, 0)\right\|_{2} .
\end{aligned}\right.
$$

Problem 1. The first problem addressed in this paper consists of establishing whether (1)-(3) is robustly exponentially stable.

Next, let us introduce the robust $\mathcal{H}_{\infty}$ norm of (1)-(3) as

$$
\gamma_{\infty}^{*}=\sup _{p \in \mathcal{P}} \gamma_{\infty}(p)
$$


where $\gamma_{\infty}(p)$ is the $\mathcal{H}_{\infty}$ norm of (1) for the fixed value $p$ of the uncertainty given by

$$
\gamma_{\infty}(p)=\sup _{u:\|u\|_{\mathcal{L}_{2}} \neq 0} \frac{\|y\|_{\mathcal{L}_{2}}}{\|u\|_{\mathcal{L}_{2}}}
$$

and $\|\cdot\|_{\mathcal{L}_{2}}$ is the $\mathcal{L}_{2}$ norm defined as

$$
\|u\|_{\mathcal{L}_{2}}=\sqrt{\sum_{k=0}^{\infty} \int_{0}^{\infty}\|u(t, k)\|_{2}^{2} d t .}
$$

Problem 2. The second problem addressed in this paper consists of determining the robust $\mathcal{H}_{\infty}$ norm of $(1)-(3)$, i.e., $\gamma_{\infty}^{*}$.

Lastly, let us introduce the robust $\mathcal{H}_{2}$ norm of (1)-(3) as

$$
\gamma_{2}^{*}=\sup _{p \in \mathcal{P}} \gamma_{2}(p)
$$

where $\gamma_{2}(p)$ is the $\mathcal{H}_{2}$ norm of (1) for the fixed value $p$ of the uncertainty given by

$$
\gamma_{2}(p)=\sqrt{\sum_{l=1}^{n_{u}} \sum_{k=0}^{\infty} \int_{0}^{\infty} g^{T}(t, k, l) g(t, k, l) d t}
$$

where $g(t, k, l)$ is the impulse response due to a Dirac impulse applied at $k=0$ to the $l$-th channel, i.e., the solution of $y(t, k)$ for null initial conditions and $u(t, k)$ given by

$$
u(t, k)= \begin{cases}\delta(t) b(l) & \text { if } k=0 \\ 0 & \text { otherwise }\end{cases}
$$

where $\delta(t)$ is the Dirac impulse and $b(l)$ is the $l$-th canonical basis vector in $\mathbb{R}^{n_{u}}$.

Problem 3. The third problem addressed in this paper consists of determining the robust $\mathcal{H}_{2}$ norm of (1)-(3), i.e., $\gamma_{2}^{*}$.

\subsection{SOS Matrix Polynomials}

Here we briefly introduce some preliminaries about SOS matrix polynomials, see [26] and references therein for details. For reasons that will become clear 
in the next sections, we consider matrix polynomials in the variables $\omega \in \mathbb{R}$ and $p \in \mathbb{R}^{q}$.

Let us start by considering a real matrix polynomial $J(\omega, p)=J(\omega, p)^{T}$. This matrix polynomial is said to be SOS if there exist real matrix polynomials $J_{i}(\omega, p), i=1, \ldots, l$, such that

$$
J(\omega, p)=\sum_{i=1}^{l} J_{i}(\omega, p)^{T} J_{i}(\omega, p) .
$$

A necessary and sufficient condition for establishing whether $J(\omega, p)$ is SOS can be obtained via an LMI feasibility test. Indeed, $J(\omega, p)$ can be expressed as

$$
J(\omega, p)=(b(\omega, p) \otimes I)^{T}(K+L(\alpha))(b(\omega, p) \otimes I)
$$

where $b(\omega, p)$ is a vector whose entries are monomials in $\omega$ and $p, K$ is a symmetric matrix satisfying

$$
J(\omega, p)=(b(\omega, p) \otimes I)^{T} K(b(\omega, p) \otimes I),
$$

$L(\alpha)$ is a linear matrix function that parametrizes the linear subspace

$$
\mathcal{L}=\left\{L=L^{T}: \quad(b(\omega, p) \otimes I)^{T} L(b(\omega, p) \otimes I)=0\right\}
$$

and $\alpha$ is a free vector. The representation (13) is known as square matrix representation (SMR) and extends the Gram matrix method for polynomials to the case of matrix polynomials. One has that $J(\omega, p)$ is SOS if and only if there exists $\alpha$ satisfying the LMI

$$
K+L(\alpha) \geq 0 .
$$

Next, let us consider a complex matrix polynomial $J(\omega, p)=J(\omega, p)^{H}$. We say that this matrix polynomial is SOS if

$$
\Phi(J(\omega, p)) \text { is } \operatorname{SOS}
$$

where

$$
\Phi(J(\omega, p))=\left(\begin{array}{cc}
\operatorname{Re}(J(\omega, p)) & \operatorname{Im}(J(\omega, p)) \\
\star & \operatorname{Re}(J(\omega, p))
\end{array}\right) .
$$




\section{Robust Exponential Stability}

Let us start by observing that, for the case of 2D mixed continuous-discretetime systems without uncertainty, a necessary condition for exponential stability is that the matrix multiplying $x_{c}(t, k)$ in the expression of $\frac{d}{d t} x_{c}(t, k)$ is Hurwitz and the matrix multiplying $x_{d}(t, k)$ in the expression of $x_{d}(t, k+1)$ is Schur. In particular, we say that $M \in \mathbb{C}^{n \times n}$ is Hurwitz if

$$
\operatorname{Re}\left(\lambda_{i}(M)\right)<0 \quad \forall i=1, \ldots, n
$$

and we say that $M \in \mathbb{C}^{n \times n}$ is Schur if

$$
\left|\lambda_{i}(M)\right|<1 \quad \forall i=1, \ldots, n .
$$

This means that, without loss of generality, we can introduce the following assumption.

Assumption 1. For all $p \in \mathcal{P}, A_{c c}(p)$ is Hurwitz and $A_{d d}(p)$ is Schur.

This assumption can be checked in various ways. One of these is the simplification of the results proposed in this section to the case of 1D systems, which has been reported in Corollary 1 in Appendix 1. Another way consists of adopting existing methods such as those mentioned in the introduction.

Let us denote with $X_{d}(s, k)$ the Laplace transform of $x_{d}(t, k)$, where $s \in \mathbb{C}$. For null initial conditions and null input, one has from (1) that

$$
X_{d}(s, k+1)=F_{1}(s, p) X_{d}(s, k)
$$

where

$$
F_{1}(s, p)=A_{d c}(p)\left(s I-A_{c c}(p)\right)^{-1} A_{c d}(p)+A_{d d}(p) .
$$

Let us express $F_{1}(s, p)$ as

$$
F_{1}(s, p)=\frac{G_{1}(s, p)}{g(s, p)}
$$

where $G_{1}(s, p)$ is a real matrix polynomial, and

$$
g(s, p)=\operatorname{det}\left(s I-A_{c c}(p)\right) .
$$

The following result provides a necessary and sufficient condition for robust exponential stability based on the eigenvalues of $F_{1}(s, p)$, and is a direct extension of the same result for the case of $2 \mathrm{D}$ mixed continuous-discretetime systems without uncertainty reported in [3] and references therein. 
Lemma 1 The system (1)-(3) is robustly exponentially stable if and only if

$$
\left|\lambda_{i}\left(F_{1}(j \omega, p)\right)\right|<1 \quad \forall i=1, \ldots, n_{d} \forall \omega \in \mathbb{R} \forall p \in \mathcal{P} .
$$

This lemma can be exploited to derive a condition for robust exponential stability of the system (1)-(3) through the use of suitable Lyapunov function candidates. To this end, let us introduce the following definition, which extends the concepts of even and odd complex matrix functions introduced in $[12]$ to the case of two variables.

Definition 1. For a complex matrix function $M(\omega, p)$, we say that $M(\omega, p)$ is even in $\omega$ if

$$
M(-\omega, p)=\overline{M(\omega, p)} \quad \forall \omega \in \mathbb{R} \forall p \in \mathbb{R}^{q}
$$

and we say that $M(\omega, p)$ is odd in $\omega$ if

$$
M(-\omega, p)=-\overline{M(\omega, p)} \quad \forall \omega \in \mathbb{R} \forall p \in \mathbb{R}^{q} .
$$

Based on Definition 1, let us define

$$
\begin{aligned}
\mathcal{M}_{\text {even }}= & \{M(\omega, p): M(\omega, p) \text { is a Hermitian matrix } \\
& \text { polynomial in } \omega \text { and } p, \text { even in } \omega\} .
\end{aligned}
$$

The following result provides a condition for robust exponential stability of (1)-(3) based on the existence of a complex Lyapunov function with polynomial dependence on $\omega$ and $p$.

Theorem 1 The system (1)-(3) is robustly exponentially stable if there exist $\zeta>0$ and $V(\omega, p) \in \mathcal{M}_{\text {even }}$ such that

$$
\forall \omega \in \mathbb{R} \forall p \in \mathcal{P}\left\{\begin{array}{l}
0 \leq V(\omega, p)-\zeta I \\
0 \leq W(\omega, p)-\zeta|g(j \omega, p)|^{2} I
\end{array}\right.
$$

where

$$
\begin{aligned}
W(\omega, p)= & |g(j \omega, p)|^{2} V(\omega, p) \\
& -G_{1}(j \omega, p)^{H} V(\omega, p) G_{1}(j \omega, p) .
\end{aligned}
$$


Moreover, if $\mathcal{P}$ is compact, this condition is not only sufficient but also necessary, and the degree of $V(\omega, p)$ can be chosen not greater than $2 d_{\text {sta }}$ where

$$
d_{s t a}=\max \left\{n_{c} n_{d}^{2}, d_{s y s}\left(\left(n_{c}+1\right) n_{d}^{2}-1\right)\right\} .
$$

Proof. " $\Leftarrow$ " Suppose that $(29)$ holds. From Assumption 1 it follows that

$$
g(j \omega, p) \neq 0 \quad \forall \omega \in \mathbb{R} \forall p \in \mathcal{P}
$$

which implies

$$
\forall \omega \in \mathbb{R} \forall p \in \mathcal{P}\left\{\begin{aligned}
0 \leq & V(\omega, p)-\zeta I \\
0 \leq & V(\omega, p)-\zeta I \\
& -F_{1}(j \omega, p)^{H} V(\omega, p) F_{1}(j \omega, p)
\end{aligned}\right.
$$

and, hence, (25) holds. Consequently, from Lemma 1 we conclude that (1)-(3) is robustly exponentially stable.

" $\Rightarrow$ " Suppose that (1)-(3) is robustly exponentially stable and that $\mathcal{P}$ is compact. From Lemma 1 one has that (25) holds. From Assumption 1 and from the fact that $G_{1}(j \omega, p)$ and $g(j \omega, p)$ are even in $\omega$, it follows that the Lyapunov equation

$$
\left\{\begin{array}{l}
\tilde{V}(\omega, p)-F_{1}(j \omega, p)^{H} \tilde{V}(\omega, p) F_{1}(j \omega, p)=I \\
p \in \mathcal{P}
\end{array}\right.
$$

has a unique solution $\tilde{V}(\omega, p)$ which satisfies

$$
\left\{\begin{array}{l}
\tilde{V}(\omega, p) \geq \varepsilon_{1} \quad \forall \omega \in \mathbb{R} \forall p \in \mathcal{P} \\
\tilde{V}(\omega, p) \text { is even in } \omega
\end{array}\right.
$$

for some $\varepsilon_{1}>0$. This Lyapunov equation can be rewritten as

$$
E(\omega, p) \tilde{v}(\omega, p)=f
$$

where $\tilde{v}(\omega, p)$ and $f$ are $n_{d}^{2} \times 1$ real vector functions that gather the real and imaginary parts of the entries of $\tilde{V}(\omega, p)$ and $I$ in their upper triangular parts, and $E(\omega, p)$ is a $n_{d}^{2} \times n_{d}^{2}$ real matrix function. Due to Assumption 1 and since the solution of the introduced Lyapunov equation is unique, one has

$$
|\operatorname{det}(E(\omega, p))| \geq \varepsilon_{2} \quad \forall \omega \in \mathbb{R} \forall p \in \mathcal{P}
$$


for some $\varepsilon_{2}>0$. Hence,

$$
\tilde{v}(\omega, p)=E(\omega, p)^{-1} f .
$$

Let us observe that $E(\omega, p)$ can be written as

$$
E(\omega, p)=\frac{E_{\text {num }}(\omega, p)}{|g(j \omega, p)|^{2}}
$$

where $E_{\text {num }}(\omega, p)$ is a real matrix polynomial. Since $|g(j \omega, p)|^{2}$ is a real polynomial of degree not greater than $2 d_{g}$ where

$$
d_{g}=n_{c} \max \left\{1, d_{\text {sys }}\right\},
$$

it follows that $E_{\text {num }}(\omega, p)$ is a real matrix polynomial of degree not greater than $2 d_{E}$ where

$$
d_{E}=\max \left\{n_{c}, d_{\text {sys }}\left(n_{c}+1\right)\right\} .
$$

Taking into account Assumption 1, it follows that

$$
\left|\operatorname{det}\left(E_{\text {num }}(\omega, p)\right)\right| \geq \varepsilon_{3} \quad \forall \omega \in \mathbb{R} \forall p \in \mathcal{P}
$$

for some $\varepsilon_{3}>0$. Hence, the inverse of $E(\omega, p)$ is

$$
E(\omega, p)^{-1}=|g(j \omega, p)|^{2} \frac{\operatorname{adj}\left(E_{\text {num }}(\omega, p)\right)}{\operatorname{det}\left(E_{\text {num }}(\omega, p)\right)}
$$

where $\operatorname{adj}\left(E_{\text {num }}(\omega, p)\right)$ is a real matrix polynomial of degree not greater than $2 d_{E}\left(n_{d}^{2}-1\right)$ and $\operatorname{det}\left(E_{\text {num }}(\omega, p)\right)$ is a real polynomial of degree not greater than $2 d_{E} n_{d}^{2}$. Hence,

$$
\begin{aligned}
\tilde{v}(\omega, p) & =|g(j \omega, p)|^{2} \frac{\operatorname{adj}\left(E_{\text {num }}(\omega, p)\right)}{\operatorname{det}\left(E_{\text {num }}(\omega, p)\right)} f \\
& =\frac{\tilde{v}_{\text {num }}(\omega, p)}{\operatorname{det}\left(E_{N}(\omega, p)\right)}
\end{aligned}
$$

where $\tilde{v}_{\text {num }}(\omega, p)$ is a real vector polynomial of degree not greater than $2 d_{\text {sta }}$. Next, let us define

$$
c(\omega, p)=\operatorname{sgn}\left(\operatorname{det}\left(E_{\text {num }}\left(\omega_{0}, p_{0}\right)\right)\right) \operatorname{det}\left(E_{\text {num }}(\omega, p)\right)
$$

where $\omega_{0} \in \mathbb{R}$ and $p_{0} \in \mathcal{P}$ are arbitrary values. Since $E_{\text {num }}(\omega, p)$ is a real matrix polynomial, one has

$$
c(\omega, p) \geq \varepsilon_{4} \quad \forall \omega \in \mathbb{R} \forall p \in \mathcal{P}
$$


for some $\varepsilon_{4}>0$. Let us define

$$
V(\omega, p)=c(\omega, p) \tilde{V}(\omega, p) .
$$

Due to Assumption 1, it follows that

$$
\forall \omega \in \mathbb{R} \forall p \in \mathcal{P}\left\{\begin{aligned}
0 \leq & V(\omega, p)-\varepsilon_{1} \varepsilon_{4} I \\
0= & |g(j \omega, p)|^{2} V(\omega, p) \\
& -G(j \omega, p)^{H} V(\omega, p) G(j \omega, p) \\
& -c(\omega, p)|g(j \omega, p)|^{2} I .
\end{aligned}\right.
$$

Moreover, $V(\omega, p)$ is even in $\omega$ because $c(\omega, p)$ and $\tilde{V}(\omega, p)$ are. Hence, (29) holds with $V(\omega, p) \in \mathcal{M}_{\text {even }}$ whose degree is not greater than $2 d_{\text {sta }}$, and $\zeta$ given by

$$
\zeta=\min \left\{\varepsilon_{1} \varepsilon_{4}, \varepsilon_{4}\right\}>0 .
$$

Theorem 1 provides a sufficient condition for robust exponential stability of (1)-(3) based on the existence of $\zeta>0$ and $V(\omega, p) \in \mathcal{M}_{\text {even }}$ satisfying (29). The complex matrix polynomial $V(\omega, p)$ defines a complex Lyapunov function with polynomial dependence on $\omega$ and $p$. Moreover, Theorem 1 also states that this condition is not only sufficient but also necessary whenever $\mathcal{P}$ is compact, and provides an upper bound on the degree of $V(\omega, p)$.

Hereafter we will show how the condition provided by Theorem 1 can be checked with an LMI feasibility test. Before presenting this result, let us introduce the following definition.

Definition 2. We say that the set $\mathcal{P}$ in (3) is strongly compact if $\mathcal{P}$ is compact and the polynomials $a_{i}(p), i=1, \ldots, n_{a}$, have even degree with their highest degree homogeneous parts having no common zeros except 0 .

The above definition introduces the notion of strongly compact sets, which will be exploited in some of the results proposed in this paper (specifically, Theorems 2, 3 and 5) in order to provide conditions that are not only sufficient but also necessary.

Theorem 2 The system (1)-(3) is robustly exponentially stable if there exist $\zeta>0$ and $V(\omega, p), T_{i}(\omega, p), U_{i}(\omega, p) \in \mathcal{M}_{\text {even }}, i=1, \ldots, n_{a}$, satisfying 
the LMI condition

$$
\left.\begin{array}{r}
X(\omega, p) \\
Y(\omega, p) \\
T_{i}(\omega, p) \forall i=1, \ldots, n_{a} \\
U_{i}(\omega, p) \forall i=1, \ldots, n_{a}
\end{array}\right\} \text { are } S O S
$$

where

$$
\left\{\begin{aligned}
X(\omega, p)= & V(\omega, p)-\zeta I-\sum_{i=1}^{n_{a}} a_{i}(p) T_{i}(\omega, p) \\
Y(\omega, p)= & W(\omega, p)-\zeta|g(j \omega, p)|^{2} I \\
& -\sum_{i=1}^{n_{a}} a_{i}(p) U_{i}(\omega, p) .
\end{aligned}\right.
$$

Moreover, if $\mathcal{P}$ is strongly compact, this condition is not only sufficient but also necessary for (1)-(3) to be robustly exponentially stable.

Proof. " $\Leftarrow$ " Suppose that $(32)$ holds. From the definition of SOS matrix polynomials in Section 2.2, the first constraint in (32) implies that

$$
X(\omega, p) \geq 0 \quad \forall \omega \in \mathbb{R} \forall p \in \mathbb{R}^{q} .
$$

Similarly, one obtains that $Y(\omega, p), T_{i}(\omega, p)$ and $U_{i}(\omega, p)$ are positive semidefinite for all $\omega \in \mathbb{R}$ for all $p \in \mathbb{R}^{q}$. Next, let $\omega_{0} \in \mathbb{R}$ and $p_{0} \in \mathcal{P}$ be arbitrary values. The positive semidefiniteness of $X(\omega, p)$ and $T_{i}(\omega, p)$ implies that

$$
\begin{aligned}
0 & \leq X\left(\omega_{0}, p_{0}\right) \\
& =V\left(\omega_{0}, p_{0}\right)-\zeta I-\sum_{i=1}^{n_{a}} a_{i}\left(p_{0}\right) T_{i}\left(\omega_{0}, p_{0}\right) \\
& \leq V\left(\omega_{0}, p_{0}\right)-\zeta I
\end{aligned}
$$

since $a_{i}\left(p_{0}\right) \geq 0$. This means that $V(\omega, p)-\zeta I \geq 0$ for all $\omega \in \mathbb{R}$ for all $p \in \mathbb{R}^{q}$. Analogously, one proves that $W(\omega, p)-\zeta|g(j \omega, p)|^{2} I \geq 0$ for all $\omega \in \mathbb{R}$ for all $p \in \mathbb{R}^{q}$. Hence, (29) holds. Lastly, $\zeta$ is positive, and therefore we can conclude from Theorem 1 that (1)-(3) is robustly exponentially stable.

" $\Rightarrow$ " Suppose that (1)-(3) is robustly exponentially stable and that $\mathcal{P}$ is strongly compact. From Theorem 1 it follows that there exist $\zeta>0$ and $V(\omega, p) \in \mathcal{M}_{\text {even }}$ of degree not greater than $2 d_{\text {sta }}$ such that (29) holds. Let us consider the first constraint in (29). This is equivalent to

$$
\Phi(V(\omega, p))-\zeta I \geq 0 \quad \forall \omega \in \mathbb{R} \forall p \in \mathcal{P} .
$$


Let us introduce the parameterized SMR of $\Phi(V(\omega, p))$ with respect to $p$ as (see Section 2.2 about the SMR)

$$
\begin{aligned}
\Phi(V(\omega, p))= & (\tilde{b}(\omega) \otimes I)^{T}(\tilde{K}(p)+\tilde{L}(\tilde{\alpha}(p))) \\
& \cdot(\tilde{b}(\omega) \otimes I)
\end{aligned}
$$

where $\tilde{b}(\omega)$ is a vector whose entries are the monomials in $\omega$ of degree less than or equal to $d_{s t a}$, and $\tilde{K}(p)+\tilde{L}(\tilde{\alpha}(p))$ is a symmetric matrix function. In particular, $\tilde{K}(p)$ is a symmetric matrix polynomial of degree not greater than $2 d_{\text {sta }}$, and $\tilde{L}(\tilde{\alpha}(p))$ is a linear matrix function of $\tilde{\alpha}(p)$, which is an arbitrary vector function of suitable size. Since $\omega$ is a scalar, the condition $\Phi(V(\omega, p))-\zeta I \geq 0$ for all $\omega \in \mathbb{R}$ for all $p \in \mathbb{R}^{q}$ is equivalent to the existence of $\tilde{\zeta}>0$ and a real vector function $\tilde{\alpha}(p)$ such that (see for instance [26])

$$
\tilde{K}(p)+\tilde{L}(\tilde{\alpha}(p))-\tilde{\zeta} I \geq 0 \quad \forall p \in \mathcal{P} .
$$

Since $\Phi(V(\omega, p))$ is continuous with respect to $p$ and $\mathcal{P}$ is compact, there exists $\hat{\zeta}>0$ and a real vector polynomial $\hat{\alpha}(p)$ such that

$$
\tilde{K}(p)+\tilde{L}(\hat{\alpha}(p))-\hat{\zeta} I \geq 0 \quad \forall p \in \mathcal{P} .
$$

Since $\mathcal{P}$ is strongly compact, it follows from Putinar's Positivstellensatz [22] that there exist $\check{\zeta}>0$ and SOS real matrix polynomials $\tilde{T}_{i}(p), i=1, \ldots, n_{a}$, such that

$$
\tilde{X}(p)=\tilde{K}(p)+\tilde{L}(\hat{\alpha}(p))-\check{\zeta} I-\sum_{i=1}^{n_{a}} a_{i}(p) \tilde{T}_{i}(p)
$$

is SOS. Hence,

$$
\hat{X}(\omega, p)=(\tilde{b}(\omega) \otimes I)^{T} \tilde{X}(p)(\tilde{b}(\omega) \otimes I)
$$

is SOS. Since the SOS real matrix polynomials $\tilde{T}_{i}(p)$ can be chosen with the same block structure of $\tilde{K}(p)$ introduced by the function $\Phi(\cdot)$, and since $V(\omega, p)$ is even in $\omega$, one has that

$$
\hat{X}(\omega, p)=\Phi\left(V(\omega, p)-\check{\zeta} I-\sum_{i=1}^{n_{a}} a_{i}(p) T_{i}(\omega, p)\right)
$$

where each $T_{i}(\omega, p)$ is even in $\omega$ and SOS. Hence, the first and the third constraints in (32) hold with $\zeta$ replaced by $\check{\zeta}$. Similarly, one proves that also 
the second and the fourth constraints in (32) hold.

Theorem 2 provides a condition based on SOS matrix polynomials for establishing the robust exponential stability of (1)-(3). Since establishing whether a complex matrix polynomials is SOS is equivalent to an LMI as explained in Section 2.2, and since $X(\omega, p)$ and $Y(\omega, p)$ are affine linear matrix functions of the decision variables $\zeta, V(\omega, p), T_{i}(\omega, p)$ and $U_{i}(\omega, p)$, it follows that the condition (32) amounts to solving an LMI feasibility test. This conversion can be done by using existing software such as [27].

The LMI condition provided by Theorem 2 is sufficient for any chosen degrees of $V(\omega, p), T_{i}(\omega, p)$ and $U_{i}(\omega, p)$, and it is also necessary for sufficiently large degrees under the assumption that $\mathcal{P}$ is strongly compact.

A simple way to choose the degrees of $V(\omega, p), T_{i}(\omega, p)$ and $U_{i}(\omega, p)$ is the following. First, one arbitrarily chooses the degree of $V(\omega, p)$, which defines the candidate complex Lyapunov function. Let us denote this degree as $2 d$, where $d$ is a nonnegative integer. Then, one chooses the degrees of $T_{i}(\omega, p)$ and $U_{i}(\omega, p)$ as the largest degrees for which $X(\omega, p)$ and $Y(\omega, p)$ have their minimum degrees. We denote the sets of $V(\omega, p), T_{i}(\omega, p)$ and $U_{i}(\omega, p)$ in $\mathcal{M}_{\text {even }}$ with degrees bounded as just described as $\mathcal{V} \mathcal{T U}(2 d)$.

In order to quantify the feasibility of (32), let us introduce the index

$$
\begin{aligned}
& \zeta^{*}=\sup _{\left(V(\omega, p), T_{i}(\omega, p), U_{i}(\omega, p)\right) \in \mathcal{V} \mathcal{T U}(2 d)} \zeta \\
& \text { s.t. }\left\{\begin{array}{l}
(32) \text { holds } \\
\operatorname{trace}\left(V\left(\omega_{0}, p_{0}\right)\right)=1
\end{array}\right.
\end{aligned}
$$

where $\omega_{0} \in \mathbb{R}$ and $p_{0} \in \mathcal{P}$ are arbitrarily chosen values. Let us observe that the condition $\operatorname{trace}\left(V\left(\omega_{0}, p_{0}\right)\right)=1$ can be introduced without loss of generality since $V(\omega, p)$ and $\zeta$ are defined up to a positive scale factor in the LMI condition provided by Theorem 2. The index $\zeta^{*}$ quantifies feasibility of the condition provided by Theorem 2 , in particular

$$
\text { (32) holds } \Longleftrightarrow \zeta^{*}>0 \text {. }
$$

Consequently, one has that

$$
\begin{gathered}
(1)-(3) \text { is robustly exponentially stable } \\
\qquad \exists: \stackrel{\mathbb{}}{\zeta^{*}>0 .}
\end{gathered}
$$

The optimization problem (34), which amounts to minimizing a linear function subject to SOS constraints and linear equations, is a convex optimization problem, in particular a semidefinite program (SDP). 


\section{Robust $\mathcal{H}_{\infty}$ Norm}

Let us denote with $U_{L}(s, k)$ and $Y_{L}(s, k)$ the Laplace transforms of $u(t, k)$ and $y(t, k)$, where $s \in \mathbb{C}$. Let us denote with $U_{L Z}(s, z)$ and $Y_{L Z}(s, z)$ the Z-transforms of $U_{L}(s, k)$ and $Y_{L}(s, k)$, where $z \in \mathbb{C}$. The transfer function from $u(t, k)$ and $y(t, k)$ is denoted by $F(s, z, p)$ and satisfies

$$
Y_{L Z}(s, z)=F(s, z, p) U_{L Z}(s, z) .
$$

Standard manipulations show that

$$
F(s, z, p)=F_{3}(s, p)\left(z I-F_{1}(s, p)\right)^{-1} F_{2}(s, p)+F_{4}(s, p)
$$

where $F_{1}(s, p)$ is given by $(22)$ and

$$
\left\{\begin{array}{l}
F_{2}(s, p)=A_{d c}(p)\left(s I-A_{c c}(p)\right)^{-1} B_{c}(p)+B_{d}(p) \\
F_{3}(s, p)=C_{c}(p)\left(s I-A_{c c}(p)\right)^{-1} A_{c d}(p)+C_{d}(p) \\
F_{4}(s, p)=C_{c}(p)\left(s I-A_{c c}(p)\right)^{-1} B_{c}(p)+D(p) .
\end{array}\right.
$$

We express $F_{i}(s, p), i=1, \ldots, 4$, as

$$
F_{i}(s, p)=\frac{G_{i}(s, p)}{g(s, p)}
$$

where $G_{i}(s, p), i=1, \ldots, 4$, are real matrix polynomials, and $g(s, p)$ is given by $(24)$.

The quantity $\gamma_{\infty}(p)$ in (7) can be written as

$$
\gamma_{\infty}(p)=\|F(\cdot, \cdot, p)\|_{L Z-\mathcal{H}_{\infty}}
$$

where $\|F(\cdot, \cdot, p)\|_{L Z-\mathcal{H}_{\infty}}$ is the Laplace-Z $\mathcal{H}_{\infty}$ norm of $F(s, z, p)$ defined as

$$
\|F(\cdot, \cdot, p)\|_{L Z-\mathcal{H}_{\infty}}=\sup _{\substack{\omega \in \mathbb{R} \\ \theta \in[-\pi, \pi]}}\left\|F\left(j \omega, e^{j \theta}, p\right)\right\|_{2} .
$$

Hence, it follows that

$$
\gamma_{\infty}(p)=\sup _{\omega \in \mathbb{R}}\|F(j \omega, \cdot, p)\|_{Z-\mathcal{H}_{\infty}}
$$

where $\|F(j \omega, \cdot, p)\|_{Z-\mathcal{H}_{\infty}}$ is the $\mathrm{Z} \mathcal{H}_{\infty}$ norm of $F(j \omega, z, p)$ defined as

$$
\|F(j \omega, \cdot, p)\|_{Z-\mathcal{H}_{\infty}}=\sup _{\theta \in[-\pi, \pi]}\left\|F\left(j \omega, e^{j \theta}, p\right)\right\|_{2} .
$$


Let us introduce the Lyapunov function candidate

$$
V_{\text {rat }}(\omega, p)=\frac{V(\omega, p)}{v(\omega)}
$$

where

$$
\left\{\begin{aligned}
V(\omega, p) & \in \mathcal{M}_{\text {even }} \\
\operatorname{deg}(V(\omega, p) & \leq 2 d
\end{aligned}\right.
$$

for a nonnegative integer $d$, and

$$
v(\omega)=\left(1+\omega^{2}\right)^{d} .
$$

Define

$$
Q(\omega, p)=\left(\begin{array}{cc}
q_{1}(\omega, p) & q_{2}(\omega, p) \\
\star & q_{3}(\omega, p)
\end{array}\right)
$$

where

$$
\left\{\begin{aligned}
q_{1}(\omega, p)= & |g(j \omega, p)|^{2} V(\omega, p) \\
& -G_{1}(j \omega, p) V(\omega, p) G_{1}(j \omega, p)^{H} \\
& -v(\omega) G_{2}(j \omega, p) G_{2}(j \omega, p)^{H} \\
q_{2}(\omega, p)= & -G_{1}(j \omega, p) V(\omega, p) G_{3}(j \omega, p)^{H} \\
& -v(\omega) G_{2}(j \omega, p) G_{4}(j \omega, p)^{H} \\
q_{3}(\omega, p)= & \xi v(\omega)|g(j \omega, p)|^{2} I \\
& -G_{3}(j \omega, p) V(\omega, p) G_{3}(j \omega, p)^{H} \\
& -v(\omega) G_{4}(j \omega, p) G_{4}(j \omega, p)^{H}
\end{aligned}\right.
$$

and $\xi \in \mathbb{R}$. Since $V(\omega, p), G_{i}(j \omega, p)$ and $g(j \omega, p)$ are even in $\omega$, it follows that also $Q(\omega, p)$ is even in $\omega$.

The following result provides an upper bound on the robust $\mathcal{H}_{\infty}$ norm of (1)-(3) via an LMI feasibility test.

Theorem 3 Let $\xi>0$. One has

$$
\sqrt{\xi}>\gamma_{\infty}^{*}
$$

if there exist $\zeta>0$ and $V(\omega, p), T_{i}(\omega, p), U_{i}(\omega, p) \in \mathcal{M}_{\text {even }}, i=1, \ldots, n_{a}$, with $\operatorname{deg}(V(\omega, p)) \leq 2 d$ satisfying the LMI condition

$$
\left.\begin{array}{r}
X(\omega, p) \\
Y_{\infty}(\omega, p) \\
T_{i}(\omega, p) \forall i=1, \ldots, n_{a} \\
U_{i}(\omega, p) \forall i=1, \ldots, n_{a}
\end{array}\right\} \text { are } S O S
$$


where $X(\omega, p)$ is as in (33) and

$$
\begin{aligned}
Y_{\infty}(\omega, p)= & Q(\omega, p)-\sum_{i=1}^{n_{a}} a_{i}(p) U_{i}(\omega, p) \\
& -\zeta v(\omega)|g(j \omega, p)|^{2} I .
\end{aligned}
$$

Moreover, if $\mathcal{P}$ is strongly compact, this condition is not only sufficient but also necessary for (50) by using a sufficiently large $d$.

Proof. " $\Leftarrow$ " Suppose that (51) holds. It follows that

$$
\forall \omega \in \mathbb{R} \forall p \in \mathbb{R}^{q}\left\{\begin{aligned}
0 & \leq X(\omega, p) \\
0 & \leq Y_{\infty}(\omega, p) \\
0 & \leq T_{i}(\omega, p) \forall i=1, \ldots, n_{a} \\
0 & \leq U_{i}(\omega, p) \forall i=1, \ldots, n_{a} .
\end{aligned}\right.
$$

This implies that

$$
Q(\omega, p) \geq \zeta v(\omega)|g(j \omega, p)|^{2} I \quad \forall \omega \in \mathbb{R} \forall p \in \mathcal{P} .
$$

Let us observe that

$$
Q(\omega, p)=v(\omega)|g(j \omega, p)|^{2} \tilde{Q}(\omega, p)
$$

where $\tilde{Q}(\omega, p)$ is obtained from $Q(\omega, p)$ replacing $q_{1}(\omega, p), q_{2}(\omega, p), q_{3}(\omega, p)$ with $\tilde{q}_{1}(\omega, p), \tilde{q}_{2}(\omega, p), \tilde{q}_{3}(\omega, p)$ given by

$$
\left\{\begin{aligned}
\tilde{q}_{1}(\omega, p)= & V_{\text {rat }}(\omega, p) \\
& -F_{1}(j \omega, p) V_{\text {rat }}(\omega, p) F_{1}(j \omega, p)^{H} \\
& -F_{2}(j \omega, p) F_{2}(j \omega, p)^{H} \\
\tilde{q}_{2}(\omega, p)= & -F_{1}(j \omega, p) V_{\text {rat }}(\omega, p) F_{3}(j \omega, p)^{H} \\
& -F_{2}(j \omega, p) F_{4}(j \omega, p)^{H} \\
\tilde{q}_{3}(\omega, p)= & \xi I-F_{3}(j \omega, p) V_{\text {rat }}(\omega, p) F_{3}(j \omega, p)^{H} \\
& -F_{4}(j \omega, p) F_{4}(j \omega, p)^{H} .
\end{aligned}\right.
$$

Since Assumption 1 implies that there exists $\varepsilon_{1}>0$ such that

$$
|g(j \omega, p)| \geq \varepsilon_{1} \quad \forall \omega \in \mathbb{R} \forall p \in \mathbb{R}^{q},
$$

and since

$$
v(\omega) \geq 1 \quad \forall \omega \in \mathbb{R},
$$

one can write

$$
\tilde{Q}(\omega, p) \geq \zeta I \quad \forall \omega \in \mathbb{R} \forall p \in \mathcal{P} .
$$


Similarly, one has the same result for $V_{\text {rat }}(\omega, p)$, and from the bounded real lemma and Schur complement it follows that

$$
\sqrt{\xi}>\|F(j \omega, \cdot, p)\|_{Z-\mathcal{H}_{\infty}} \quad \forall \omega \in \mathbb{R} \forall p \in \mathcal{P} .
$$

From (6) and (43), this implies that (50) holds.

" $\Rightarrow$ " Suppose that (50) holds and that $\mathcal{P}$ is strongly compact. This implies that there exist $\tilde{\zeta}>0$ and a Hermitian matrix function $\tilde{V}(\omega, p)$ even in $\omega$ such that

$$
\forall \omega \in \mathbb{R} \forall p \in \mathbb{R}^{q}\left\{\begin{array}{l}
0 \leq \tilde{V}(\omega, p)-\tilde{\zeta} I \\
0 \leq \tilde{Q}(\omega, p)-\tilde{\zeta} I
\end{array}\right.
$$

where $\tilde{Q}(\omega, p)$ is obtained as in the first part of the proof by replacing $V_{\text {rat }}(\omega, p)$ with $\tilde{V}(\omega, p)$. The limit as $\omega$ tends to $\infty$ of $\tilde{V}(\omega, p)$ does exist, i.e.,

$$
\lim _{\omega \rightarrow \infty} \tilde{V}(\omega, p)=\tilde{V}_{\infty}(p)
$$

for some symmetric matrix function $\tilde{V}_{\infty}(p)$. Let us define

$$
\tilde{V}_{0}(\omega, p)=\operatorname{Re}(\tilde{V}(\omega, p)) .
$$

Since $\tilde{V}(\omega, p)$ is an Hermitian matrix function even in $\omega$, it follows that $\tilde{V}_{0}(\omega, p)$ can be rewritten as

$$
\tilde{V}_{0}(\omega, p)=\tilde{V}_{1}\left(\omega^{2}, p\right)
$$

where $\tilde{V}_{1}(\omega, p)$ is a symmetric matrix function. Let us define

$$
\left\{\begin{array}{l}
m_{1}(\psi)=\frac{\psi}{1-\psi} \\
m_{2}(\omega)=\frac{\omega^{2}}{1+\omega^{2}}
\end{array}\right.
$$

and

$$
\tilde{V}_{2}(\psi, p)=\tilde{V}_{1}\left(m_{1}(\psi), p\right)
$$

It follows that

$$
\begin{aligned}
& \forall \omega \in \mathbb{R} \forall p \in \mathbb{R}^{q} \exists \psi=m_{2}(\omega) \in[0,1): \\
& \tilde{V}_{1}\left(\omega^{2}, p\right)=\tilde{V}_{2}(\psi, p) \text {. }
\end{aligned}
$$

Since $\tilde{V}_{2}(\psi, p)$ is continuous and

$$
\lim _{\psi \rightarrow 1} \tilde{V}_{2}(\psi, p)=\tilde{V}_{\infty}(p),
$$


it follows that $\tilde{V}_{2}(\psi, p)$ can be approximated arbitrarily well over $[0,1]$ by a symmetric matrix polynomial $\tilde{V}_{3}(\psi, p)$. Hence, let us define

$$
\tilde{V}_{4}(\omega, p)=\tilde{V}_{3}\left(m_{2}(\omega), p\right) .
$$

It follows that $\tilde{V}_{4}(\omega, p)$ is a symmetric rational function that approximates arbitrarily well the continuous function $\tilde{V}_{0}(\omega, p)$. Moreover, since $\tilde{V}_{4}(\omega, p)$ is even in $\omega$, it follows that $\tilde{V}_{4}(\omega, p)$ has the form

$$
\tilde{V}_{4}(\omega, p)=\frac{\tilde{V}_{5}(\omega, p)}{v(\omega)}
$$

where $v(\omega)$ is as in $(47)$, and $\tilde{V}_{5}(\omega, p)$ is a symmetric matrix polynomial of degree $2 d$ even in $\omega$.

Next, let us define

$$
\tilde{V}_{6}(\omega, p)=\operatorname{Im}(\tilde{V}(\omega, p)) .
$$

It follows that $\tilde{V}_{6}(\omega, p)$ can be rewritten as

$$
\tilde{V}_{6}(\omega, p)=\omega \tilde{V}_{7}\left(\omega^{2}, p\right)
$$

where $\tilde{V}_{7}(\omega, p)$ is a skew-symmetric matrix function. One has that $\tilde{V}_{7}\left(m_{1}(\psi), p\right)$ can be approximated arbitrarily well by a skew-symmetric matrix polynomial $\tilde{V}_{8}(\psi, p)$ over $[0,1]$, and hence

$$
\tilde{V}_{9}(\omega, p)=j \omega \tilde{V}_{8}\left(m_{2}(\omega), p\right)
$$

is a skew-symmetric rational function that approximates arbitrarily well $\tilde{V}_{6}(\omega, p)$. In particular,

$$
\tilde{V}_{9}(\omega, p)=\frac{\tilde{V}_{10}(\omega, p)}{v(\omega)}
$$

where $\tilde{V}_{10}(\omega, p)$ is a skew-symmetric matrix polynomial of degree $2 d$ such that $j \tilde{V}_{10}(\omega, p)$ is even in $\omega$.

Lastly, let us define $V_{\text {rat }}(\omega, p)$ as in (45) with $V(\omega, p)$ given by

$$
V(\omega, p)=\tilde{V}_{5}(\omega, p)+j \tilde{V}_{10}(\omega, p)
$$

and let $\tilde{Q}(\omega, p)$ be defined as in the first part of the proof with such a $V_{\text {rat }}(\omega, p)$. Due to the continuity of $\tilde{Q}(\omega, p)$ with respect to $V_{\text {rat }}(\omega, p)$, it follows that the degree $2 d$ can be chosen such that $V_{\text {rat }}(\omega, p) \geq \tilde{\zeta} I$ and $\tilde{Q}(\omega, p) \geq \tilde{\zeta} I$ for all $\omega \in \mathbb{R}$ for all $p \in \mathcal{P}$ for some $\tilde{\zeta}>0$. This implies that 
$V(\omega, p) \geq \tilde{\zeta} v(\omega) I$ and $Q(\omega, p) \geq \tilde{\zeta} v(\omega)|g(j \omega)|^{2} I$ for all $\omega \in \mathbb{R}$ for all $p \in \mathcal{P}$. Proceeding as in the second part of Theorem 2, we conclude that there exist $\zeta>0$ and $T_{i}(\omega, p), U_{i}(\omega, p) \in \mathcal{M}_{\text {even }}$ such that (51) hold.

Theorem 3 provides an LMI condition for establishing upper bounds on $\gamma_{\infty}^{*}$. Let us observe that $V_{\text {rat }}(\omega, p)$ in (45) defines a complex Lyapunov function candidate with rational dependence in $\omega$ and polynomial dependence in $p$.

The LMI condition provided by Theorem 3 is sufficient for any chosen degrees of $V(\omega, p), T_{i}(\omega, p)$ and $U_{i}(\omega, p)$, and it is also necessary for sufficiently large degrees under the assumption that $\mathcal{P}$ is strongly compact.

The degrees of $V(\omega, p), T_{i}(\omega, p)$ and $U_{i}(\omega, p)$ in Theorem 3 can be chosen analogously to what done for Theorem 2. In particular, we denote with $\mathcal{V} \mathcal{T} \mathcal{U}_{\infty}(2 d)$ the set $\mathcal{V} \mathcal{T U}(2 d)$ analogously obtained for Theorem 3.

Let us observe that the complex Lyapunov function candidate introduced in (45) might not be the only one able to provide a necessary and sufficient condition in Theorem 3. Indeed, it is possible that the denominator $v(\omega)$ can be defined differently from (47), provided that $v(\omega)$ is a positive polynomial of degree $2 d$.

Let us define

$$
\hat{\gamma}_{\infty}=\sqrt{\hat{\xi}}
$$

where $\hat{\xi}$ is the solution of the SDP

$$
\hat{\xi}=\inf _{\left(V(\omega, p), T_{i}(\omega, p), U_{i}(\omega, p)\right) \in \mathcal{V} \mathcal{T} \mathcal{U}_{\infty}(2 d)} \xi
$$

s.t. (51) holds.

It follows that

$$
\hat{\gamma}_{\infty} \geq \gamma_{\infty}^{*} .
$$

Once that the upper bound $\hat{\gamma}_{\infty}$ has been obtained, a question arises: is this upper bound tight? The following result answers this question.

Theorem 4 Suppose that $\hat{\gamma}_{\infty}<\infty$. Then,

$$
\hat{\gamma}_{\infty}=\gamma_{\infty}^{*}
$$

if at least one of the following two sub-conditions holds: 
1) there exists $\hat{\omega} \in \mathbb{R}$ and $\hat{p} \in \mathcal{P}$ such that

$$
\|F(j \hat{\omega}, \cdot, \hat{p})\|_{Z-\mathcal{H}_{\infty}}=\hat{\gamma}_{\infty}
$$

and

$$
\operatorname{det}\left(\hat{Y}_{\infty}(\hat{\omega}, \hat{p})\right)=0
$$

where $\hat{Y}_{\infty}(\omega, p)$ is $Y_{\infty}(\omega, p)$ evaluated for the optimal values of the decision variables in (51);

2) there exists $\hat{p} \in \mathcal{P}$ such that

$$
\lim _{\omega \rightarrow \infty}\|F(j \omega, \cdot, \hat{p})\|_{Z-\mathcal{H}_{\infty}}=\hat{\gamma}_{\infty}
$$

Moreover, if $\mathcal{P}$ is compact, this condition is not only sufficient but also necessary.

Proof. " $\Leftarrow$ " Suppose that $(57)-(58)$ or $(59)$ holds. Then, it follows that $\hat{\gamma}_{\infty} \leq \gamma_{\infty}^{*}$ since $\gamma_{\infty}^{*}$ is the supremum of $\|F(j \omega, \cdot, p)\|_{Z-\mathcal{H}_{\infty}}$ for $\omega \in \mathbb{R}$ and $p \in \mathcal{P}$, while Theorem 3 guarantees that $\hat{\gamma}_{\infty} \geq \gamma_{\infty}^{*}$. Therefore, (56) holds.

" $\Rightarrow$ " Suppose that (56) holds and that $\mathcal{P}$ is compact. There are two possibilities. The first is that there exist $\hat{\omega} \in \mathbb{R}$ and $\hat{p} \in \mathcal{P}$ such that

$$
\gamma_{\infty}^{*}=\|F(j \hat{\omega}, \cdot, \hat{p})\|_{Z-\mathcal{H}_{\infty}} .
$$

It follows that (58) holds. In fact, supposing for contradiction that (58) does not hold, from the fact that $\Phi(S(\omega, p))$ is SOS it would follow that

$$
\Phi\left(\hat{Y}_{\infty}(\hat{\omega}, \hat{p})\right)>0
$$

hence implying that (51) holds with $\xi<\hat{\xi}$, which is impossible for definition of $\hat{\xi}$. The second possibility is that there exists $\hat{p} \in \mathcal{P}$ such that (59) holds.

Theorem 4 provides a necessary and sufficient condition for establishing whether the found upper bound $\hat{\gamma}_{\infty}$ is tight. This condition consists of two sub-conditions.

1) In order to check the first sub-condition, one determines the pairs $(\hat{\omega}, \hat{p})$ that satisfy $(58)$. This can be done as follows. Since $\hat{Y}_{\infty}(\omega, p)$ is a SOS matrix polynomial, from Section 2.2 one can write

$$
\hat{Y}_{\infty}(\omega, p)=(b(\omega, p) \otimes I)^{T} K_{1}(b(\omega, p) \otimes I)
$$


where $K_{1}$ is a symmetric positive semidefinite matrix. Hence, (58) holds if and only if

$$
(b(\hat{\omega}, \hat{p}) \otimes I) \in \operatorname{ker}\left(K_{1}\right) .
$$

The search for the pairs $(\hat{\omega}, \hat{p})$ that satisfy the above condition can be addressed by pivoting operations that reduce the problem to finding the roots of a polynomial in a single variable, see [28] for details. Once that these pairs have been found, one trivially checks whether (57) holds for any of them.

2) In order to check the second sub-condition of Theorem 4, one can adopt a strategy similar to the one just described in this section by computing the robust $\mathcal{H}_{\infty}$ norm of the system described by $F_{1}(s, p), \ldots, F_{4}(s, p)$ for $s$ that tends to $\infty$, see Appendix 2 .

\section{Robust $\mathcal{H}_{2}$ Norm}

The quantity $\gamma_{2}(p)$ in (10) can be written as

$$
\gamma_{2}(p)=\|F(\cdot, \cdot, p)\|_{L Z-\mathcal{H}_{2}}
$$

where $\|F(\cdot, \cdot, p)\|_{L Z-\mathcal{H}_{2}}$ is the Laplace-Z $\mathcal{H}_{2}$ norm of $F(s, z, p)$ defined as

$$
\frac{1}{2 \pi} \sqrt{\int_{-\infty}^{\infty} \int_{-\pi}^{\pi} \operatorname{trace}\left(F\left(j \omega, e^{j \theta}, p\right)^{H} F\left(j \omega, e^{j \theta}, p\right)\right) d \theta d \omega .}
$$

Hence, it follows that

$$
\gamma_{2}(p)=\sqrt{\frac{1}{2 \pi} \int_{-\infty}^{\infty}\|F(j \omega, \cdot, p)\|_{Z-\mathcal{H}_{2}}^{2} d \omega}
$$

where $\|F(j \omega, \cdot, p)\|_{Z-\mathcal{H}_{2}}$ is the $\mathrm{Z} \mathcal{H}_{2}$ norm of $F(j \omega, \cdot, p)$ defined as

$$
\frac{\|F(j \omega, \cdot, p)\|_{Z-\mathcal{H}_{2}}=}{\sqrt{\frac{1}{2 \pi} \int_{-\pi}^{\pi} \operatorname{trace}\left(F\left(j \omega, e^{j \theta}, p\right)^{H} F\left(j \omega, e^{j \theta}, p\right)\right) d \theta} .}
$$

Therefore, a necessary condition for $\gamma_{2}(p)$ to be finite is

$$
\lim _{\omega \rightarrow \infty} F\left(j \omega, e^{j \theta}, p\right)=0 .
$$


The idea to construct upper bounds on $\gamma_{2}$ is to make use of the Lyapunov function candidate defined by

$$
V_{\text {rat }}(\omega, p)=\frac{V(\omega, p)}{v(\omega)}
$$

where

$$
\left\{\begin{aligned}
V(\omega, p) & \in \mathcal{M}_{\text {even }} \\
\operatorname{deg}(V(\omega, p) & \leq 2 d-2
\end{aligned}\right.
$$

for a positive integer $d$, and $v(\omega)$ is given by (47). Let us define

$$
\begin{aligned}
R(\omega, p)= & |g(j \omega, p)|^{2} V(\omega, p)-v(\omega) G_{2}(\omega, p) G_{2}(\omega, p)^{H} \\
& -G_{1}(\omega, p) V(\omega, p) G_{1}(\omega, p)^{H} .
\end{aligned}
$$

The following result provides an upper bound on the $\mathcal{H}_{2}$ norm of the system (1) via an LMI feasibility test.

Theorem 5 One has

$$
\sqrt{\xi}>\gamma_{2}
$$

if there exist $\zeta>0, V(\omega, p), T_{i}(\omega, p), U_{i}(\omega, p) \in \mathcal{M}_{\text {even }}$, and polynomials $u_{i}(p), i=1, \ldots, n_{a}$, with $\operatorname{deg}(V(\omega, p)) \leq 2 d-2$ satisfying the LMI condition

$$
\left.\begin{array}{r}
X(\omega, p) \\
Y_{2}(\omega, p) \\
y_{2}(p) \\
T_{i}(\omega, p) \forall i=1, \ldots, n_{a} \\
U_{i}(\omega, p) \forall i=1, \ldots, n_{a} \\
u_{i}(p) \forall i=1, \ldots, n_{a}
\end{array}\right\} \text { are } S O S
$$

where $X(\omega, p)$ is as in (33) and

$$
\left\{\begin{aligned}
Y_{2}(\omega, p)= & R(\omega, p)-\sum_{i=1}^{n_{a}} a_{i}(p) U_{i}(\omega, p) \\
& -\zeta v(\omega)|g(j \omega, p)|^{2} I \\
y_{2}(p)= & \xi-\psi(p)-\sum_{i=1}^{n_{a}} a_{i}(p) u_{i}(p)
\end{aligned}\right.
$$

where

$$
\psi(p)=\frac{1}{2 \pi} \int_{-\infty}^{\infty} \phi(\omega, p) d \omega
$$


and

$$
\begin{aligned}
\phi(\omega, p)= & \operatorname{trace}\left(F_{3}(\omega, p) V_{\text {rat }}(\omega, p) F_{3}(\omega, p)^{H}\right. \\
& \left.+F_{4}(\omega, p) F_{4}(\omega, p)^{H}\right) .
\end{aligned}
$$

Moreover, if $\mathcal{P}$ is strongly compact, this condition is not only sufficient but also necessary for (70) by using a sufficiently large $d$.

Proof. " $\Leftarrow$ " Suppose that $(71)$ holds. It follows that

$$
R(\omega, p) \geq \zeta v(\omega)|g(j \omega, p)|^{2} I \quad \forall \omega \in \mathbb{R} \forall p \in \mathcal{P} .
$$

Let us observe that

$$
R(\omega, p)=v(\omega)|g(j \omega, p)|^{2} \tilde{R}(\omega, p)
$$

where

$$
\begin{aligned}
\tilde{R}(\omega, p)= & V_{\text {rat }}(\omega, p)-F_{2}(\omega, p) F_{2}(\omega, p)^{H} \\
& -F_{1}(\omega, p) V_{\text {rat }}(\omega, p) F_{1}(\omega, p)^{H} .
\end{aligned}
$$

Analogously to the proof of Theorem 3, it follows that

$$
\tilde{R}(\omega, p) \geq \zeta I \quad \forall \omega \in \mathbb{R} \forall p \in \mathcal{P} .
$$

Similarly, one has the same result for $V_{\text {rat }}(\omega, p)$, hence implying that

$$
\phi(\omega, p)>\|F(j \omega, \cdot, p)\|_{Z-\mathcal{H}_{2}}^{2} \quad \forall \omega \in \mathbb{R} \forall p \in \mathcal{P} .
$$

Since

$$
\xi \geq \psi(p) \quad \forall p \in \mathcal{P},
$$

it follows that

$$
\xi>\sqrt{\frac{1}{2 \pi} \int_{-\infty}^{\infty}\|F(j \omega, \cdot, p)\|_{Z-\mathcal{H}_{2}}^{2} d \omega} \quad \forall p \in \mathcal{P},
$$

that is, (70).

" $\Rightarrow$ " Suppose that (70) holds and that $\mathcal{P}$ is strongly compact. Reverting the first part of the proof, and proceeding as in the second part of the proof of Theorem 3, one shows that there exist $\zeta>0, V(\omega, p), T_{i}(\omega, p), U_{i}(\omega, p) \in$ $\mathcal{M}_{\text {even }}$ and polynomials $u_{i}(p)$ with $\operatorname{deg}(V(\omega, p)) \leq 2 d-2$ such that (71) holds for a sufficiently large $d$.

Theorem 5 provides an LMI condition for establishing upper bounds on $\gamma_{2}^{*}$. Let us observe that $V_{\text {rat }}(\omega, p)$ in (67) is strictly proper contrary to the one in (45) introduced to determine $\gamma_{\infty}^{*}$. 
The LMI condition provided by Theorem 5 is sufficient for any chosen degrees of $V(\omega, p), T_{i}(\omega, p), U_{i}(\omega, p)$ and $u_{i}(p)$, and it is also necessary for sufficiently large degrees under the assumption that $\mathcal{P}$ is strongly compact.

The degrees of $V(\omega, p), T_{i}(\omega, p), U_{i}(\omega, p)$ and $u_{i}(p)$ in Theorem 5 can be chosen analogously to what done for Theorem 2 . In particular, we denote with $\mathcal{V} \mathcal{T} \mathcal{U}_{2}(2 d)$ the set $\mathcal{V} \mathcal{T U}(2 d)$ analogously obtained for Theorem 5.

Let us define

$$
\hat{\gamma}_{2}=\sqrt{\hat{\xi}}
$$

where $\hat{\xi}$ is the solution of the SDP

$$
\begin{aligned}
\hat{\xi}= & \inf _{\substack{\xi, \zeta>0 \\
\left(V(\omega, p), T_{i}(\omega, p), U_{i}(\omega, p), u_{i}(p)\right) \in \mathcal{V} \mathcal{T} \mathcal{U}_{2}(2 d)}} \zeta \\
& \text { s.t. }(71) \text { holds. }
\end{aligned}
$$

It follows that

$$
\hat{\gamma}_{2} \geq \gamma_{2}^{*}
$$

\section{Examples}

In this section we present some illustrative examples of the proposed results. The SDPs (34), (54) and (76) are solved with the toolbox SeDuMi [29] for Matlab on a personal computer with Windows 8, Intel Core i7, $3.4 \mathrm{GHz}, 8$ GB RAM. The SDP (34) is solved with the choice $\omega_{0}=1$ and $p_{0}$ equal to the center of $\mathcal{P}$.

\subsection{Example 1}

Let us start by considering a variant of the vehicle platoon model in [5] described by

$$
\left\{\begin{aligned}
\frac{d}{d t} x_{c_{1}}(t, k)= & x_{c_{2}}(t, k) \\
m \frac{d}{d t} x_{c_{2}}(t, k)= & -K_{p} x_{c_{1}}(t, k)-\left(b+(1+h) K_{p}\right) x_{c_{2}}(t, k) \\
& -K_{i} x_{c_{3}}(t, k)+K_{p}\left(x_{d_{1}}(t, k)+x_{d_{2}}(t, k)\right) \\
\frac{d}{d t} x_{c_{3}}(t, k)= & x_{c_{1}}(t, k)+(1+h) x_{c_{2}}(t, k)-x_{d_{1}}(t, k) \\
& -x_{d_{2}}(t, k) \\
x_{d_{1}}(t, k+1)= & \beta x_{c_{1}}(t, k) \\
x_{d_{2}}(t, k+1)= & x_{c_{2}}(t, k)
\end{aligned}\right.
$$


where $x_{c_{1}}, x_{c_{2}} \in \mathbb{R}$ are the position and velocity of the car, $x_{c_{3}} \in \mathbb{R}$ is the state of the proportional-integral controller, $x_{d_{1}}, x_{d_{2}} \in \mathbb{R}$ are the position and velocity of the preceding car, $K_{p}, K_{i} \in \mathbb{R}$ are gains, $m, b \in \mathbb{R}$ are the mass and friction of the car, $h \in \mathbb{R}$ is time headway, and $\beta \in \mathbb{R}$ is a coefficient indicating the fraction of the position of the preceding car to be used. We consider the plausible values

$$
\left\{\begin{array}{l}
K_{p}=2, K_{i}=2, m=2, b=0.6, \beta=0.9 \\
h \in[1,3] .
\end{array}\right.
$$

By defining $p=h$, the system can be described by (1)-(3) with

$$
\left\{\begin{array}{c}
A_{c c}(p)=\left(\begin{array}{ccc}
0 & 1 & 0 \\
-1 & -0.3-1.5 p & -1 \\
1 & 1+p & 0
\end{array}\right), A_{c d}(p)=\left(\begin{array}{cc}
0 & 0 \\
1 & 1 \\
-1 & -1
\end{array}\right) \\
A_{d c}(p)=\left(\begin{array}{ccc}
0.9 & 0 & 0 \\
0 & 1 & 0
\end{array}\right), A_{d d}(p)=\left(\begin{array}{cc}
0 & 0 \\
0 & 0
\end{array}\right) \\
n_{a}=1, a_{1}(p)=-3+4 p-p^{2} .
\end{array}\right.
$$

The problem consists of establishing whether this system is robustly exponentially stable, i.e., Problem 1.

First, we verify that Assumption 1 holds, i.e., that $A_{c c}(p)$ is Hurwitz and $A_{d d}(p)$ is Schur for all $p \in \mathcal{P}$. The fact that $A_{d d}(p)$ is Schur for all $p \in \mathcal{P}$ is obvious since this matrix is null. Concerning $A_{c c}(p)$, we verify that this matrix is Hurwitz for all $p \in \mathcal{P}$ by using Corollary 1, in particular (78) holds with symmetric matrix polynomials $\tilde{V}(p), \tilde{T}_{i}(p), \tilde{U}_{i}(p)$ of degree 0 .

Second, we calculate the index $\zeta^{*}$ in $(34)$. We simply choose $2 d=0$, finding

$$
\zeta^{*}=0.181
$$

which proves robust exponential stability according to Theorem 2. The number of LMI scalar variables in the SDP (34) is 232, and its computational time is less than 1 second. The found Lyapunov function is described by

$$
V(\omega, p)=\left(\begin{array}{cc}
0.500 & 0.482 \\
\star & 0.500
\end{array}\right)
$$




\subsection{Example 2}

Here we consider (1)-(3) with

$$
\left\{\begin{array}{l}
A_{c c}(p)=\left(\begin{array}{cc}
0 & 1 \\
-4 & -2
\end{array}\right), A_{c d}(p)=\left(\begin{array}{c}
-0.5-0.3 p_{2} \\
0.4 p_{1}
\end{array}\right) \\
A_{d c}(p)=\left(\begin{array}{cc}
2 & 0.5
\end{array}\right), A_{d d}(p)=0.5 \\
B_{c}(p)=\left(\begin{array}{c}
0 \\
1+p_{1}-p_{2}
\end{array}\right), B_{d}(p)=1 \\
C_{c}(p)=\left(\begin{array}{cc}
0 & -1
\end{array}\right), C_{d}(p)=1 \\
D(p)=1, \mathcal{P}=\left\{p \in \mathbb{R}^{2}:\|p\|_{2} \leq 1\right\} .
\end{array}\right.
$$

The problem consists of determining the robust $\mathcal{H}_{\infty}$ norm of this system, i.e., Problem 2.

In order to solve this problem, we solve (53)-(54) expressing $\mathcal{P}$ as in (3) with $a_{1}(p)=1-p_{1}^{2}-p_{2}^{2}$. We simply choose $2 d=0$, finding the upper bound

$$
\hat{\gamma}_{\infty}=5.586
$$

This upper bound can be improved by increasing $d$, indeed with $2 d=2$ we find

$$
\hat{\gamma}_{\infty}=4.563 \text {. }
$$

The found Lyapunov function is described by $V_{\text {rat }}(\omega, p)$ in (45) with

$$
\begin{aligned}
V(\omega, p)= & 2.774 p_{1}^{2}-6.148 p_{1} p_{2}-8.251 p_{1}+2.949 p_{2}^{2} \\
& +8.079 p_{2}+3.725 \omega^{2}+6.731 .
\end{aligned}
$$

The number of LMI scalar variables in the SDP (54) is 856, and its computational time is 4.2 seconds.

Next, let us use Theorem 4 to establish whether the found upper bound is tight. We find that (58) holds with

$$
\hat{\omega}=2.627, \quad \hat{p}=\left(\begin{array}{c}
-0.657 \\
0.754
\end{array}\right) .
$$

Moreover, for such values of $\hat{\omega}$ and $\hat{p}$, one has that (57) holds. Consequently, from Theorem 4 we conclude that $\hat{\gamma}_{\infty}$ is tight, i.e., $\gamma_{\infty}^{*}=4.563$. 


\subsection{Example 3}

Lastly, we consider (1)-(3) with

$$
\left\{\begin{array}{l}
A_{c c}(p)=\left(\begin{array}{cc}
-1 & 1 \\
1 & -2
\end{array}\right), A_{c d}(p)=\left(\begin{array}{cc}
-0.7 & -0.4 \\
0.6 & 0.6
\end{array}\right) \\
A_{d c}(p)=\left(\begin{array}{cc}
0 & 1 \\
-1+p & 0
\end{array}\right), A_{d d}(p)=\left(\begin{array}{cc}
0.3 & -0.5 \\
0.5 & 0.3
\end{array}\right) \\
B_{c}(p)=\left(\begin{array}{cc}
1 & 0 \\
1 & p
\end{array}\right), B_{d}(p)=\left(\begin{array}{ll}
0 & 0 \\
0 & 0
\end{array}\right) \\
C_{c}(p)=\left(\begin{array}{cc}
0 & 0.3 \\
0 & 0.5 p
\end{array}\right), C_{d}(p)=\left(\begin{array}{ll}
1 & 0 \\
0 & 1
\end{array}\right) \\
D(p)=\left(\begin{array}{ll}
0 & 0 \\
0 & 0
\end{array}\right), \mathcal{P}=[-1,1] .
\end{array}\right.
$$

The problem consists of determining the robust $\mathcal{H}_{2}$ norm of this system, i.e., Problem 3.

In order to solve this problem, we solve (75)-(76) expressing $\mathcal{P}$ as in (3) with $a_{1}(p)=1-p^{2}$. By using different values of $2 d$, we find the following upper bounds:

$$
\left\{\begin{array}{lll}
2 d=2 & \Rightarrow & \hat{\gamma}_{2}=\infty \\
2 d=4 & \Rightarrow & \hat{\gamma}_{2}=7.144 \\
2 d=6 & \Rightarrow & \hat{\gamma}_{2}=6.138 .
\end{array}\right.
$$

In the case $2 d=6$, the number of LMI scalar variables in the SDP (76) is 1340 , and its computational time is 16.2 seconds. The found Lyapunov function is described by $V_{\text {rat }}(\omega, p)$ in (67) with

$$
V(\omega, p)=\left(\begin{array}{cc}
v_{1} & v_{2} \\
\star & v_{3}
\end{array}\right)
$$

where

$$
\left\{\begin{aligned}
v_{1}= & -0.338 p^{4}-3.151 p^{3}+23.745 p^{2} \omega^{2}+1.681 p^{2} \\
& +82.392 p \omega^{2}+1.988 p+5.625 \omega^{4}+87.338 \omega^{2} \\
& +26.131 \\
v_{2}= & -0.86 p^{4}+1.54 p^{3}+9.717 p^{2} \omega^{2}-3.618 p^{2} \\
& +26.57 p \omega^{2}+10.733 p-1.697 \omega^{4}+15.936 \omega^{2} \\
& -11.267+j\left(-35.591 p^{3} \omega+18.191 p^{2} \omega\right. \\
& \left.+12.279 p \omega^{3}+0.758 p \omega-6.885 \omega^{3}+20.274 \omega\right) \\
v_{3}= & 3.313 p^{4}-2.653 p^{3}-49.545 p^{2} \omega^{2}+15.322 p^{2} \\
& +31.209 p \omega^{2}-66.084 p+10.102 \omega^{4}+96.923 \omega^{2} \\
& +84.463
\end{aligned}\right.
$$




\section{Conclusion}

This paper has proposed LMI conditions for establishing robust exponential stability and for determining the robust $\mathcal{H}_{\infty}$ and $\mathcal{H}_{2}$ norms of $2 \mathrm{D}$ mixed continuous-discrete-time systems whose coefficients are polynomial functions of an uncertain vector constrained into a semialgebraic set. The proposed LMI conditions are based on the introduction of complex Lyapunov functions depending polynomially or rationally on a frequency and polynomially on the uncertainty. It has been shown that these LMI conditions are sufficient for any chosen degrees of the complex Lyapunov functions, and also necessary for sufficiently large degrees of these functions under some mild assumptions on the set of admissible uncertainties.

Several directions can be explored in future works. In particular, one could investigate the possibility of generalizing the results proposed in this paper to marginally stable systems. Another possibility could be the extension of the proposed results to systems with time-varying uncertainties. Also, one could explore the extension of the proposed results to the presence of communications constraints in the system, which are typical of networked control systems such as those considered in [30,31]. A last possibility could be the extension to the design of robust controllers.

\section{Acknowledgements}

The author would like to thank the Associate Editor and the Reviewers for their useful comments. This work is supported in part by the Research Grants Council of Hong Kong under Grant HKU711213E.

\section{Appendix 1}

The following corollary shows how Theorem 2 can be specialized to provide an LMI condition for establishing whether Assumption 1 holds.

Corollary 1 The matrix $A_{c c}(p)$ is Hurwitz (respectively, $A_{d d}(p)$ is Schur) for all $p \in \mathcal{P}$ if there exist $\zeta>0$ and symmetric matrix polynomials $\tilde{V}(p), \tilde{T}_{i}(p), \tilde{U}_{i}(p)$, 
$i=1, \ldots, n_{a}$, satisfying the $L M I$ condition

$$
\left.\begin{array}{r}
\tilde{X}(p) \\
\tilde{Y}(p) \\
\tilde{T}_{i}(p) \forall i=1, \ldots, n_{a} \\
\tilde{U}_{i}(p) \forall i=1, \ldots, n_{a}
\end{array}\right\} \text { are } S O S
$$

where

$$
\left\{\begin{array}{l}
\tilde{X}(p)=\tilde{V}(p)-\zeta I-\sum_{i=1}^{n_{a}} a_{i}(p) \tilde{T}_{i}(p) \\
\tilde{Y}(p)=\tilde{W}(p)-\zeta I-\sum_{i=1}^{n_{a}} a_{i}(p) \tilde{U}_{i}(p)
\end{array}\right.
$$

and $\tilde{W}(p)=-\tilde{V}(p) A_{c c}(p)-A_{c c}(p)^{T} \tilde{V}(p)$ (respectively, $\tilde{W}(p)=\tilde{V}(p)-$ $\left.A_{d d}(p) \tilde{V}(p) A_{d d}(p)^{T}\right)$. Moreover, if $\mathcal{P}$ is strongly compact, this condition is not only sufficient but also necessary.

Proof. Analogous to the proof of Theorem 2 by removing the dependence on $\omega$ of the matrices.

\section{Appendix 2}

Let $\tilde{Q}(p)$ be the matrix polynomial obtained from $Q(\omega, p)$ in (48) by replacing $q_{1}(\omega, p), q_{2}(\omega, p), q_{3}(\omega, p)$ with $\tilde{q}_{1}(p), \tilde{q}_{2}(p), \tilde{q}_{3}(p)$ defined as

$$
\left\{\begin{array}{l}
\tilde{q}_{1}(p)=\tilde{V}(p)-A_{d d}(p) \tilde{V}(p) A_{d d}(p)^{T}-B_{d}(p) B_{d}(p)^{T} \\
\tilde{q}_{2}(p)=-A_{d d}(p) \tilde{V}(p) C_{d}(p)^{T}-B_{d}(p) D(p)^{T} \\
\tilde{q}_{3}(p)=\xi I-C_{d}(p) \tilde{V}(p) C_{d}(p)^{T}-D(p) \tilde{V}(p) D(p)^{T}
\end{array}\right.
$$

where $\tilde{V}(p)$ is a symmetric matrix polynomial.

Let us define

$$
\tilde{\gamma}_{\#}=\sqrt{\tilde{\xi}}
$$

where $\tilde{\xi}$ is the solution of the SDP

$$
\begin{aligned}
\tilde{\xi}= & \inf _{\substack{\xi, \zeta>0 \\
\tilde{V}(p), \tilde{T}_{i}(p), \tilde{U}_{i}(p) \in \mathcal{V} \mathcal{T} \mathcal{U}_{\#}(2 d)}} \xi \\
& \text { s.t. }(78) \text { holds with } \tilde{W}(p) \text { replaced by } \tilde{Q}(p)
\end{aligned}
$$


where $\mathcal{V} \mathcal{T} \mathcal{U}_{\#}(2 d)$ is the set of symmetric matrix polynomials $\tilde{V}(p), \tilde{T}_{i}(p), \tilde{U}_{i}(p)$ analogous to $\mathcal{V} \mathcal{T} \mathcal{U}_{\infty}(2 d)$.

The following corollary shows how Theorem 4 can be specialized to provide a strategy for establishing whether the second sub-condition of this theorem holds.

Corollary 2 Any $\hat{p} \in \mathcal{P}$ that satisfies (59) also satisfies

$$
\operatorname{det}\left(\tilde{Y}_{\infty}(\hat{p})\right)=0
$$

where $\tilde{Y}_{\infty}(p)$ is $\tilde{Y}(p)$ evaluated for the optimal values of the decision variables in $(82)$.

Proof. Analogous to the proof of Theorem 4 by removing the dependence on $\omega$ of the matrices.

Corollary 2 can be used to check the second sub-condition of Theorem 4 as follows. First, one determines the values of $\hat{p}$ that satisfy (83). This can be done by expressing $\tilde{Y}_{\infty}(p)$, which is a SOS matrix polynomial, as

$$
\tilde{Y}_{\infty}(p)=(b(p) \otimes I)^{T} K_{2}(b(p) \otimes I)
$$

where $K_{2}$ is a symmetric positive semidefinite matrix. Hence, (83) holds if and only if

$$
(b(\hat{p}) \otimes I) \in \operatorname{ker}\left(K_{2}\right) .
$$

The search for the values of $\hat{p}$ that satisfy the above condition can be addressed by pivoting operations that reduce the problem to finding the roots of a polynomial in a single variable, see [28] for details. Once that these values have been found, one trivially checks whether (59) holds for any of them.

\section{References}

[1] R. P. Roesser. A discrete state-space model for linear image processing. IEEE Transactions on Automatic Control, 20(1):1-10, 1975.

[2] E. Fornasini and G. Marchesini. Doubly-indexed dynamical systems: State-space models and structural properties. Mathematical Systems Theory, 12:59-72, 1978. 
[3] E. Rogers and D. H. Owens. Stability Analysis for Linear Repetitive Processes, volume 175 of Lecture Notes in Control And Information Sciences Series. Springer, 1992.

[4] E. Fornasini and M. E. Valcher. Recent developments in 2D positive system theory. International Journal of Applied Mathematics and Computer Science, 7(4):713-735, 1997.

[5] S. Knorn and R. H. Middleton. Stability of two-dimensional linear systems with singularities on the stability boundary using LMIs. IEEE Transactions on Automatic Control, 58(10):2579-2590, 2013.

[6] H. Kar and V. Singh. Stability of 2-D systems described by the Fornasini-Marchesini first model. IEEE Transactions on Signal Processing, 51(6):1675-1676, 2003.

[7] K. Galkowski, W. Paszke, E. Rogers, S. Xu, and J. Lam. Stability and control of differential linear repetitive processes using an LMI setting. IEEE Transactions on Circuits and Systems II: Analog and Digital Signal Processing, 50(9):662-666, 2003.

[8] D. Bouagada and P. Van Dooren. On the stability of 2D state-space models. Numerical Linear Algebra with Applications, 20(2):198-207, 2013.

[9] G. Chesi and R. H. Middleton. Necessary and sufficient LMI conditions for stability and performance analysis of 2D mixed continuous-discretetime systems. IEEE Transactions on Automatic Control, 59(4):9961007, 2014.

[10] W. Paszke, K. Galkowski, E. Rogers, and J. Lam. $H_{2}$ and mixed $H_{2} / H_{\infty}$ stabilization and disturbance attenuation for differential linear repetitive processes. IEEE Transactions on Circuits and Systems I: Regular Papers, 55(9):2813-2826, 2008.

[11] W. Paszke, E. Rogers, and K. Galkowski. $H_{2} / H_{\infty}$ output informationbased disturbance attenuation for differential linear repetitive processes. International Journal of Robust and Nonlinear Control, 21(17):1981-1993, 2011.

[12] G. Chesi and R. H. Middleton. H-infinity and H-two norms of 2D mixed continuous-discrete-time systems via rationally-dependent complex Lyapunov functions. IEEE Transactions on Automatic Control, 60(10):2614-2625, 2015. 
[13] X. Li, H. Gao, and C. Wang. Generalized Kalman-Yakubovich-Popov lemma for 2-D FM LSS model. IEEE Transactions on Automatic Control, 57(12):3090-3103, 2012.

[14] X. Li, J. Lam, H. Gao, and Y. Gu. A frequency-partitioning approach to stability analysis of two-dimensional discrete systems. Multidimensional Systems and Signal Processing, 26(1):67-93, 2015.

[15] B. Aguirre, C. Ibarra, and R. Suarez. Sufficient algebraic conditions for stability of cones of polynomials. Systems and Control Letters, 46(4):255-263, 2002.

[16] P.-A. Bliman. A convex approach to robust stability for linear systems with uncertain scalar parameters. SIAM Journal on Control and Optimization, 42(6):2016-2042, 2004.

[17] G. Chesi. Establishing stability and instability of matrix hypercubes. Systems and Control Letters, 54(4):381-388, 2005.

[18] C. W. Scherer and C. W. J. Hol. Matrix sum-of-squares relaxations for robust semi-definite programs. Mathematical Programming Series B, 107(1-2):189-211, 2006.

[19] R. C. L. F. Oliveira and P. L. D. Peres. Parameter-dependent LMIs in robust analysis: Characterization of homogeneous polynomially parameter-dependent solutions via LMI relaxations. IEEE Transactions on Automatic Control, 52(7):1334-1340, 2007.

[20] G. Chesi. Sufficient and necessary LMI conditions for robust stability of rationally time-varying uncertain systems. IEEE Transactions on Automatic Control, 58(6):1546-1551, 2013.

[21] B. Aguirre, R. Garcia-Sosa, H. Leyva, J. Solis-Daun, and F. A. Carrillo. Conditions for the schur stability of segments of polynomials of the same degree. Boletin de la Sociedad Matematica Mexicana, 21(2):309-321, 2015 .

[22] M. Putinar. Positive polynomials on compact semi-algebraic sets. Ind. Univ. Math., 42:969-984, 1993.

[23] G. Chesi and R. H. Middleton. On the robust stability of 2D mixed continuous-discrete-time systems with uncertainty. In American Control Conference, pages 4967-4972, Portland, USA, 2014. 
[24] G. Chesi. On the robust $H_{\infty}$ norm of $2 \mathrm{D}$ mixed continuous-discretetime systems with uncertainty. In IEEE Conference on Decision and Control, pages 5985-5990, Los Angeles, USA, 2014.

[25] L. Pandolfi. Exponential stability of 2-D systems. Systems and Control Letters, 4(6):381-385, 1984.

[26] G. Chesi. LMI techniques for optimization over polynomials in control: a survey. IEEE Transactions on Automatic Control, 55(11):2500-2510, 2010 .

[27] S. Prajna, A. Papachristodoulou, and P. A. Parrilo. SOSTOOLS: a general purpose sum of squares programming solver. In IEEE Conference on Decision and Control, Las Vegas, Nevada, 2002.

[28] G. Chesi, A. Garulli, A. Tesi, and A. Vicino. Homogeneous Polynomial Forms for Robustness Analysis of Uncertain Systems. Springer, 2009.

[29] J. F. Sturm. Using SeDuMi 1.02, a MATLAB toolbox for optimization over symmetric cones. Optimization Methods and Software, 11-12:625653, 1999.

[30] F. Liu, H. Gao, J. Qiu, S. Yin, J. Fan, and T. Chai. Networked multirate output feedback control for setpoints compensation and its application to rougher flotation process. IEEE Transactions on Industrial Electronics, 61(1):460-468, 2013.

[31] T. Wang, H. Gao, and J. Qiu. A combined adaptive neural network and nonlinear model predictive control for multirate networked industrial process control. IEEE Transactions on Neural Networks and Learning Systems, 27(2):416-425, 2016. 